\begin{tabular}{|c|c|c|}
\hline 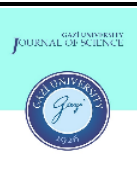 & $\begin{array}{c}\text { Gazi University } \\
\text { Journal of Science }\end{array}$ & 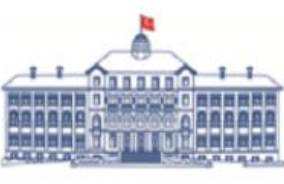 \\
\hline & http://dergipark.gov.tr/gujs & \\
\hline
\end{tabular}

\title{
A Voronovskaja-Type Theorem for a Kind of Durrmeyer-Bernstein-Stancu Operators
}

\author{
Ulku DINLEMEZ KANTAR ${ }^{1, *(1)}$, Gizem ERGELEN ${ }^{2}$ (D) \\ ${ }^{I}$ Gazi University, Faculty of Science, Department of Mathematics, Teknikokullar, 06500, Ankara, Turkey \\ ${ }^{2}$ Yukselen College, Mamak Campus, Cengizhan Mahallesi, Dogukent Cd., 06480 Mamak/Ankara, Turkey
}

\section{Highlights}

-A Durrmeyer variant of Bernstein-Stancu operators is studied.

-These operators are introduced with shifted knots.

-Asymptotic behavior of these operators is examined by using Voronovskaja-type theorem.

\section{Article Info}

Received: $16 / 01 / 2019$

Accepted: 25/03/2019

\section{Keywords}

Durrmeyer type operators

Bernstein-Stancu type operators

Voronovskaja-type theorem

\section{Abstract}

In this paper, we study on a Durrmeyer variant of Bernstein-Stancu operators. We give a Voronovskaja-type theorem for these type operators.

\section{INTRODUCTION}

In [1], for $f \in C[0,1]$, the Bernstein polynomials $\left(B_{n} f\right)$ are defined by

$B_{n}(f, x):=\sum_{k=0}^{n} f\left(\frac{k}{n}\right) b_{n k}(x), n \in \mathbb{N}$

where $b_{n k}(x):=\left(\begin{array}{l}n \\ k\end{array}\right) x^{k}(1-x)^{n-k}, k=0,1, \ldots, n$.

In [2], Durrmeyer introduced modified Bernstein polynomials to approximate Lebesgue integrable function on $[0,1]$, then he motivated the following integral modification of Bernstein polynomials

$D_{n}(f, x):=(n+1) \sum_{k=0}^{n} b_{n k}(x) \int_{0}^{1} f(t) b_{n k}(t) d t$.

In [3], the authors introduced the following new generalized Bernstein-Stancu type operators with shifted knots:

$\tilde{B}_{n}^{\alpha, \beta}(f, x):=\left(\frac{n+\beta_{2}}{n}\right)^{n} \sum_{k=0}^{n} f\left(\frac{k+\alpha_{1}}{n+\beta_{1}}\right) T_{n k}(x)$,

where $\in A_{n}:=\left[\frac{\alpha_{2}}{n+\beta_{2}}, \frac{n+\alpha_{2}}{n+\beta_{2}}\right]$, and $T_{n k}(x):=\left(\begin{array}{l}n \\ k\end{array}\right)\left(x-\frac{\alpha_{2}}{n+\beta_{2}}\right)^{k}\left(\frac{n+\alpha_{2}}{n+\beta_{2}}-x\right)^{n-k}$, 
$k=0,1, \ldots n$ with $\alpha_{k}, \beta_{k}, k=1,2$ positive numbers satisfying $0 \leq \alpha_{1} \leq \beta_{1}, 0 \leq \alpha_{2} \leq \beta_{2}$. Apparently, substituting $\alpha_{1}=\alpha_{2}=\beta_{2}=\beta_{1}=0$ in (2) one finds the classical Bernstein operators in (1), substituting $\alpha_{2}=\beta_{2}=0$ in (2), one obtains the Bernstein-Stancu operators introduced by Stancu in [4]

$B_{n, \alpha, \beta}(f, x):=\sum_{k=0}^{n} f\left(\frac{k+\alpha_{1}}{n+\beta_{1}}\right) T_{n k}(x)$.

In [5], for $f \in C[0,1]$, authors defined a Durrmeyer variant of Bernstein-Stancu operators

$S_{n}^{\alpha, \beta}(f, x):=\left(\frac{n+\beta_{2}}{n}\right)^{2 n+1} \sum_{k=0}^{n} T_{n k}(x)(n+1) \int_{A_{n}} T_{n k}(t) f\left(\frac{n t+\alpha_{1}}{n+\beta_{1}}\right) d t$,

$A_{n}:=\left[\frac{\alpha_{2}}{n+\beta_{2}}, \frac{n+\alpha_{2}}{n+\beta_{2}}\right]$, and $T_{n k}(x):=\left(\begin{array}{l}n \\ k\end{array}\right)\left(x-\frac{\alpha_{2}}{n+\beta_{2}}\right)^{k}\left(\frac{n+\alpha_{2}}{n+\beta_{2}}-x\right)^{n-k}, k=0,1, \ldots n$

with $\alpha_{k}, \beta_{k}, k=1,2$ positive numbers satisfying $0 \leq \alpha_{1} \leq \beta_{1}, 0 \leq \alpha_{2} \leq \beta_{2}$. They obtained the direct and converse results of approximation by these operators. In [6-11], many authors have studied some special cases of the operators $S_{n}^{\alpha, \beta}(f, x)$.

In this manuscript, we give a Voronovskaja-type theorem for the operators defined in (3).

\section{MAIN RESULT}

In this section, we give some lemmas in order to prove our main result. For the sake of shortness, throughout the paper we use the following notations

$H_{S}(n)=\sum_{i=2}^{s}(n+i), \quad G_{\beta_{i}}(n)=\left(n+\beta_{i}\right)$ for $i=1,2$.

Now, we give the following lemma which is proved in [5].

Lemma 1. [5] We have the following equality

$\int_{\frac{\alpha_{2}}{n+\beta_{2}}}^{\frac{n+\alpha_{2}}{n+\beta_{2}}} T_{n k}(t) d t=\left(\frac{n}{n+\beta_{2}}\right)^{n+1} \frac{1}{(n+1)}, \quad k=0,1,2 \ldots n$.

Lemma 2. Let $e_{j}=t^{j}, j=0,1,2,3,4$, we obtain

(i) $S_{n}^{\alpha, \beta}\left(e_{0}, x\right)=1$,

(ii) $S_{n}^{\alpha, \beta}\left(e_{1}, x\right)=\frac{n^{2}}{G_{\beta_{1}}(n) G_{\beta_{2}}(n)} x+\frac{n^{2}+2 n \alpha_{2}}{G_{\beta_{2}}(n) H_{2}(n)}+\frac{\alpha_{1}}{G_{\beta_{1}}(n)}$,

(iii) $S_{n}^{\alpha, \beta}\left(e_{2}, x\right)=\frac{n^{4}-n^{3}}{\left[G_{\beta_{1}}(n)\right]^{2} H_{3}(n)} x^{2}+\left\{\frac{4 n^{4}+8 n^{3} \alpha_{2}}{\left[G_{\beta_{1}}(n)\right]^{2} G_{\beta_{2}}(n) H_{3}(n)}+\frac{2 n^{2} \alpha_{1}}{\left[G_{\beta_{1}}(n)\right]^{2} H_{2}(n)}\right\} x$ $+\frac{2 n^{4}-2 \alpha_{2} n^{4}-2 \alpha_{2}^{2} n^{3}+6 n^{3} \alpha_{2}+6 n^{2} \alpha_{2}^{2}}{\left[G_{\beta_{1}}(n)\right]^{2}\left[G_{\beta_{2}}(n)\right]^{2} H_{3}(n)}+\frac{2 n^{2} \alpha_{1}+4 n \alpha_{1} \alpha_{2}}{\left[G_{\beta_{1}}(n)\right]^{2} G_{\beta_{2}}(n) H_{2}(n)}+\frac{\alpha_{1}^{2}}{\left[G_{\beta_{1}}(n)\right]^{2}}$,

(iv) $S_{n}^{\alpha, \beta}\left(e_{3}, x\right)=\frac{n^{6}-3 n^{5}-2 n^{4}}{\left[G_{\beta_{1}}(n)\right]^{3} H_{4}(n)} x^{3}+\left\{\frac{9 n^{6}+18 n^{5} \alpha_{2}-9 n^{5}-18 n^{4} \alpha_{2}}{\left[G_{\beta_{1}}(n)\right]^{3} G_{\beta_{2}}(n) H_{4}(n)}+\frac{3 n^{4} \alpha_{1}-3 n^{3} \alpha_{1}}{\left[G_{\beta_{1}}(n)\right]^{3} H_{3}(n)}\right\} x^{2}$ $+\left\{\frac{18 n^{6}-9 n^{6} \alpha_{2}-6 n^{5} \alpha_{2}^{2}+54 n^{5} \alpha_{2}+66 n^{4} \alpha_{2}^{2}}{\left[G_{\beta_{1}}(n)\right]^{3}\left[G_{\beta_{2}}(n)\right]^{2} H_{4}(n)}+\frac{12 n^{4} \alpha_{1}+24 n^{3} \alpha_{1} \alpha_{2}}{\left[G_{\beta_{1}}(n)\right]^{3} G_{\beta_{2}}(n) H_{3}(n)}+\frac{3 n^{2} \alpha_{1}^{2}}{\left[G_{\beta_{1}}(n)\right]^{3} H_{2}(n)}\right\} x$ 


$$
\begin{aligned}
& +\frac{3 n^{6} \alpha_{2}^{2}-12 n^{6} \alpha_{2}+6 n^{6}-24 n^{5} \alpha_{2}^{2}+24 n^{5} \alpha_{2}-24 n^{4} \alpha_{2}^{3}+36 n^{4} \alpha_{2}^{2}+24 n^{3} \alpha_{2}^{3}}{\left[G_{\beta_{1}}(n)\right]^{3}\left[G_{\beta_{2}}(n)\right]^{3} H_{4}(n)} \\
& +\frac{6 n^{4} \alpha_{1}-6 n^{4} \alpha_{1} \alpha_{2}-6 n^{3} \alpha_{1} \alpha_{2}^{2}+18 n^{3} \alpha_{1} \alpha_{2}+18 n^{2} \alpha_{1} \alpha_{2}^{2}}{\left[G_{\beta_{1}}(n)\right]^{3}\left[G_{\beta_{2}}(n)\right]^{2} H_{3}(n)}+\frac{3 n^{2} \alpha_{1}^{2}+6 n \alpha_{1}^{2} \alpha_{2}}{\left[G_{\beta_{1}}(n)\right]^{3} G_{\beta_{2}}(n) H_{2}(n)}+\frac{\alpha_{1}^{3}}{\left[G_{\beta_{1}}(n)\right]^{3}}, \\
& \text { (v) } S_{n}^{\alpha, \beta}\left(e_{4}, x\right)=\frac{n^{8}-6 n^{7}+11 n^{6}-6 n^{5}}{\left[G_{\beta_{1}}(n)\right]^{4} H_{5}(n)} x^{4}+\left\{\frac{24 n^{7} \alpha_{2}-4 n^{8} \alpha_{2}-44 n^{6} \alpha_{2}+24 n^{5} \alpha_{2}+16 n^{8}-48 n^{7}+32 n^{6}}{\left[G_{\beta_{1}}(n)\right]^{4} G_{\beta_{2}}(n) H_{5}(n)}\right. \\
& \left.+\frac{\left.4 n^{7} \alpha_{2}-12 n^{6} \alpha_{2}+8 n^{5} \alpha_{2}\right)(n+5)+\left(4 n^{4} \alpha_{1}-12 n^{5} \alpha_{1}+8 n^{4} \alpha_{1}\right) G_{\beta_{2}}(n)(n+5)}{\left[G_{\beta_{1}}(n)\right]^{4} G_{\beta_{2}}(n) H_{5}(n)}\right\} x^{3} \\
& +\left\{\frac{6 n^{8} \alpha_{2}^{2}-36 n^{7} \alpha_{2}^{2}+66 n^{6} \alpha_{2}^{2}-36 n^{5} \alpha_{2}^{2}-48 n^{8} \alpha_{2}+144 n^{7} \alpha_{2}-96 n^{6} \alpha_{2}+72 n^{8}-72 n^{7}}{\left[G_{\beta_{1}}(n)\right]^{4}\left[G_{\beta_{2}}(n)\right]^{2} H_{5}(n)}\right. \\
& +\frac{\left(-12 n^{7} \alpha_{2}^{2}+36 n^{6} \alpha_{2}^{2}-24 n^{5} \alpha_{2}^{2}+36 n^{7} \alpha_{2}-36 n^{6} \alpha_{2}\right)(n+5)+\left(6 n^{6} \alpha_{2}^{2}-6 n^{5} \alpha_{2}^{2}\right)(n+5)(n+4)}{\left[G_{\beta_{1}}(n)\right]^{4}\left[G_{\beta_{2}}(n)\right]^{2} H_{5}(n)} \\
& \left.+\frac{\left(36 n^{6} \alpha_{1}+72 n^{5} \alpha_{1} \alpha_{2}-36 n^{5} \alpha_{1}-74 n^{4} \alpha_{1} \alpha_{2}\right)\left(n+\beta_{2}\right)(n+5)+\left(6 n^{4} \alpha_{1}^{2}-6 n^{3} \alpha_{1}^{2}\right)\left(n+\beta_{2}\right)^{2}(n+5)(n+4)}{\left[G_{\beta_{1}}(n)\right]^{4}\left[G_{\beta_{2}}(n)\right]^{2} H_{5}(n)}\right\} x^{2} \\
& +\left\{\frac{-4 n^{8} \alpha_{2}^{3}+24 n^{7} \alpha_{2}^{3}-44 n^{6} \alpha_{2}^{3}+24 n^{5} \alpha_{2}^{3}+48 n^{8} \alpha_{2}^{2}-144 n^{7} \alpha_{2}^{2}+96 n^{6} \alpha_{2}^{2}-144 n^{8} \alpha_{2}+144 n^{7} \alpha_{2}+96 n^{4}}{\left[G_{\beta_{1}}(n)\right]^{4}\left[G_{\beta_{2}}(n)\right]^{3} H_{5}(n)}\right. \\
& +\frac{\left(12 n^{7} \alpha_{2}^{3}-36 n^{6} \alpha_{2}^{3}+24 n^{5} \alpha_{2}^{3}-72 n^{7} \alpha_{2}^{2}+72 n^{6} \alpha_{2}^{2}+72 n^{7} \alpha_{2}\right)(n+5)}{\left[G_{\beta_{1}}(n)\right]^{4}\left[G_{\beta_{2}}(n)\right]^{3} H_{5}(n)} \\
& +\frac{\left(-12 n^{6} \alpha_{2}^{3}+12 n^{5} \alpha_{2}^{3}+24 n^{6} \alpha_{2}^{2}\right)(n+5)(n+4)+4 n^{4} \alpha_{2}^{3} F_{5}(n)}{\left[G_{\beta_{1}}(n)\right]^{4}\left[G_{\beta_{2}}(n)\right]^{3} H_{5}(n)} \\
& +\frac{-36 n^{6} \alpha_{1} \alpha_{2}+72 n^{6} \alpha_{1}+216 n^{5} \alpha_{1} \alpha_{2}-24 n^{5} \alpha_{1} \alpha_{2}^{2}+264 n^{4} \alpha_{1} \alpha_{2}^{2}}{\left[G_{\beta_{1}}(n)\right]^{4}\left[G_{\beta_{2}}(n)\right]^{2} H_{4}(n)}+\frac{24 n^{4} \alpha_{1}^{2}+48 n^{3} \alpha_{1}^{2} \alpha_{2}}{\left[G_{\beta_{1}}(n)\right]^{4} G_{\beta_{2}}(n) H_{3}(n)} \\
& \left.+\frac{4 n^{2} \alpha_{1}^{3}}{\left[G_{\beta_{1}}(n)\right]^{4} H_{3}(n)}\right\} x \\
& +\frac{n^{8} \alpha_{2}^{4}-6 n^{7} \alpha_{2}^{4}+11 n^{6} \alpha_{2}^{4}-6 n^{5} \alpha_{2}^{4}-16 n^{8} \alpha_{2}^{3}+48 n^{7} \alpha_{2}^{3}-32 n^{6} \alpha_{2}^{3}+72 n^{8} \alpha_{2}^{2}-72 n^{7} \alpha_{2}^{2}}{\left[G_{\beta_{1}}(n)\right]^{4}\left[G_{\beta_{2}}(n)\right]^{4} H_{5}(n)} \\
& +\frac{24 n^{6}-96 n^{8} \alpha_{2}}{\left[G_{\beta_{1}}(n)\right]^{4}\left[G_{\beta_{2}}(n)\right]^{4} H_{5}(n)}+\frac{\left(-4 n^{7} \alpha_{2}^{4}+12 n^{6} \alpha_{2}^{4}-8 n^{5} \alpha_{2}^{4}+36 n^{7} \alpha_{2}^{3}-36 n^{6} \alpha_{2}^{3}-72 n^{7} \alpha_{2}^{2}+24 n^{7} \alpha_{2}\right)}{\left[G_{\beta_{1}}(n)\right]^{4}\left[G_{\beta_{2}}(n)\right]^{4} H_{5}(n)} \\
& +\frac{\left(6 n^{2} \alpha_{2}^{4}-6 n \alpha_{2}^{4}-24 n^{2} \alpha_{2}^{3}+12 n^{2} \alpha_{2}^{2}\right)(n+5)(n+4)}{\left[G_{\beta_{1}}(n)\right]^{4}\left[G_{\beta_{2}}(n)\right]^{4} H_{5}(n)}+\frac{\left(4 \alpha_{2}^{3}-3 \alpha_{2}^{4}\right) F_{5}(n)}{\left[G_{\beta_{1}}(n)\right]^{4}\left[G_{\beta_{2}}(n)\right]^{4} H_{5}(n)} \\
& +\frac{12 n^{6} \alpha_{1} \alpha_{2}^{2}-48 n^{6} \alpha_{1} \alpha_{2}+24 n^{6} \alpha_{1}-96 n^{5} \alpha_{1} \alpha_{2}^{2}-96 n^{5} \alpha_{1} \alpha_{2}-96 n^{4} \alpha_{1} \alpha_{2}^{3}+144 n^{4} \alpha_{1} \alpha_{2}^{2}+96 n^{3} \alpha_{1} \alpha_{2}^{3}}{\left[G_{\beta_{1}}(n)\right]^{4}\left[G_{\beta_{2}}(n)\right]^{3} H_{4}(n)} \\
& +\frac{12 n^{4} \alpha_{1}^{2}-12 n^{4} \alpha_{1}^{2} \alpha_{2}-12 n^{3} \alpha_{1}^{2} \alpha_{2}^{2}+36 n^{2} \alpha_{1}^{2} \alpha_{2}+36 n^{2} \alpha_{1}^{2} \alpha_{2}^{2}}{\left[G_{\beta_{1}}(n)\right]^{4}\left[G_{\beta_{2}}(n)\right]^{2} H_{3}(n)}+\frac{4 n^{2} \alpha_{1}^{3}+8 n \alpha_{1}^{3} \alpha_{2}}{\left[G_{\beta_{1}}(n)\right]^{4} G_{\beta_{2}}(n) H_{2}(n)}+\frac{\alpha_{1}^{4}}{\left[G_{\beta_{1}}(n)\right]^{4}} \text {. }
\end{aligned}
$$

Proof. Using equality (4) in the Durrmeyer variant of Bernstein-Stancu operators (3) for $j=0$, we get 


$$
\begin{aligned}
S_{n}^{\alpha, \beta}\left(e_{0}, x\right) & =\left(\frac{n+\beta_{2}}{n}\right)^{2 n+1} \sum_{k=0}^{n} T_{n k}(x)(n+1) \int_{A_{n}} T_{n k}(t) d t \\
& =\left(\frac{n+\beta_{2}}{n}\right)^{n} \sum_{k=0}^{n} T_{n k}(x) \\
& =1 .
\end{aligned}
$$

So the proof of $(i)$ is finished. Using the direct computation, we obtain (ii) as follows

$$
\begin{aligned}
S_{n}^{\alpha, \beta}\left(e_{1}, x\right)= & \left(\frac{n+\beta_{2}}{n}\right)^{2 n+1} \sum_{k=0}^{n} T_{n k}(x)(n+1) \int_{A_{n}} T_{n k}(t)\left(\frac{n t+\alpha_{1}}{n+\beta_{1}}\right) d t \\
= & \left(\frac{n+\beta_{2}}{n}\right)^{2 n+1} \frac{n}{n+\beta_{1}} \sum_{k=0}^{n} T_{n k}(x)(n+1)\left(\begin{array}{l}
n \\
k
\end{array}\right) \int_{A_{n}}\left(t-\frac{\alpha_{2}}{n+\beta_{2}}\right)^{k}\left(\frac{n+\alpha_{2}}{n+\beta_{2}}-t\right)^{n-k} t d t \\
& +\left(\frac{n+\beta_{2}}{n}\right)^{n} \frac{\alpha_{1}}{n+\beta_{1}} \sum_{k=0}^{n} T_{n k}(x) .
\end{aligned}
$$

If we take $t=u+\frac{\alpha_{2}}{n+\beta_{2}}$ in the last equality, then we get

$$
\begin{aligned}
S_{n}^{\alpha, \beta}\left(e_{1}, x\right)= & \left(\frac{n+\beta_{2}}{n}\right)^{2 n+1} \frac{n}{n+\beta_{1}} \sum_{k=0}^{n} T_{n k}(x)(n+1) \\
& \times\left(\begin{array}{l}
n \\
k
\end{array}\right)\left\{\int_{0}^{\frac{n}{n+\beta_{2}}} u^{k+1}\left(\frac{n}{n+\beta_{2}}-u\right)^{n-k} d u+\frac{\alpha_{2}}{n+\beta_{2}} \int_{0}^{\frac{n}{n+\beta_{2}}} u^{k}\left(\frac{n}{n+\beta_{2}}-u\right) d u\right\}, \\
S_{n}^{\alpha, \beta}\left(e_{1}, x\right)= & \left(\frac{n+\beta_{2}}{n}\right)^{2 n+1} \frac{n}{n+\beta_{1}} \sum_{k=0}^{n}\left(\begin{array}{l}
n \\
k
\end{array}\right)\left(x-\frac{\alpha_{2}}{n+\beta_{2}}\right)^{k}\left(\frac{n+\alpha_{2}}{n+\beta_{2}}-x\right)^{n-k}(n+1) \\
& \times\left\{\left(\frac{n}{n+\beta_{2}}\right)^{n+2} \frac{k+1}{(n+1)(n+2)}+\frac{\alpha_{2}}{n+\beta_{2}} \frac{1}{n+1}\left(\frac{n}{n+\beta_{2}}\right)^{n+1}\right\} \\
S_{n}^{\alpha, \beta}\left(e_{1}, x\right)= & \frac{n^{2}}{G_{\beta_{1}}(n) G_{\beta_{2}}(n)} x+\frac{n^{2}+2 n \alpha_{2}}{G_{\beta_{2}}(n) H_{2}(n)}+\frac{\alpha_{1}}{G_{\beta_{1}}(n)} .
\end{aligned}
$$

Thus, we have the proof of (ii). Finally, we apply the same process in (ii), as a result, we get (iii), (iv), and (v) easily.

Now, we give the following lemma for using the Voronovskaja-type theorem.

Lemma 3. Let $\alpha_{k}, \beta_{k}, \mathrm{k}=1,2$ be positive numbers such that $0 \leq \alpha_{1} \leq \beta_{1}, 0 \leq \alpha_{2} \leq \beta_{2}$.

We obtain the following limits 
(i) $\lim _{n \rightarrow \infty} n S_{n}^{\alpha, \beta}\left((t-x)^{2} ; x\right)=-2 x^{2}+2 x$,

(ii) $\lim _{n \rightarrow \infty} n^{2} S_{n}^{\alpha, \beta}\left((t-x)^{4} ; x\right)=28 x^{4}-24 x^{3}+\left(12 \alpha_{2}+12\right) x^{2}$.

Proof. (i) From Lemma 2, we get

$$
\begin{aligned}
& \lim _{n \rightarrow \infty} n S_{n}^{\alpha, \beta}\left((t-x)^{2} ; x\right)=\lim _{n \rightarrow \infty}\left[\left\{\frac{-2 n^{4}+n^{3}\left(6+{\beta_{1}}^{2}+4 \beta_{1}\right)+n^{2}\left(5{\beta_{1}}^{2}+12 \beta_{1}\right)+6 \beta_{1}{ }^{2} n}{\left[G_{\beta_{1}}(n)\right]^{2} H_{3}(n)}\right\} x^{2}\right. \\
& +\frac{2 n^{5}+n^{4}\left(4 \alpha_{2}+4 \alpha_{1}-2 \alpha_{1} \beta_{1}-2 \beta_{1}-6\right)+n^{3}\left(-12 \alpha_{1}-12 \alpha_{2}-10 \alpha_{1} \beta_{1}-4 \alpha_{1} \beta_{2}-4 \alpha_{2} \beta_{1}-2 \alpha_{1} \beta_{1} \beta_{2}-6 \beta_{1}\right)}{\left[G_{\beta_{1}}(n)\right]^{2}\left[G_{\beta_{2}}(n)\right]^{2} H_{3}(n)} \\
& \left.+\frac{n^{2}\left(-12 \alpha_{1} \beta_{1}-12 \alpha_{1} \beta_{2}-12 \alpha_{2} \beta_{1}-10 \alpha_{1} \beta_{1} \beta_{2}\right)}{\left[G_{\beta_{1}}(n)\right]^{2}\left[G_{\beta_{2}}(n)\right]^{2} H_{3}(n)}+\frac{-12 n \alpha_{1} \beta_{1} \beta_{2}}{\left[G_{\beta_{1}}(n)\right]^{2}\left[G_{\beta_{2}}(n)\right]^{2} H_{3}(n)}\right\} x \\
& +\frac{n^{5}\left(4 \alpha_{2}^{2}-12 \alpha_{2}+\alpha_{1}^{2}+2 \alpha_{1}+2\right)+n^{4}\left(10 \alpha_{2}^{2}+6 \alpha_{2}+5 \alpha_{1}^{2}+6 \alpha_{1}+5 \alpha_{1}^{2} \beta_{2}+2 \alpha_{1} \alpha_{2}+2 \alpha_{1} \beta_{2}\right.}{\left[G_{\beta_{1}}(n)\right]^{2}\left[G_{\beta_{2}}(n)\right]^{2} H_{3}(n)} \\
& \left.+\frac{n^{3}\left(6 \alpha_{2}^{2}+6 \alpha_{1}^{2}+10 \alpha_{1}^{2} \beta_{2}+\alpha_{1}^{2} \beta_{2}{ }^{2}+12 \alpha_{1} \alpha_{2}+6 \alpha_{1} \beta_{2}+4 \alpha_{1} \alpha_{2} \beta_{2}\right)+n^{2}\left(5 \alpha_{1}^{2} \beta_{2}{ }^{2}+12 \alpha_{1}^{2} \beta_{2}+12 \alpha_{1} \alpha_{2} \beta_{2}\right)+6 \alpha_{1}^{2} \beta_{2}{ }^{2} n}{\left[G_{\beta_{1}}(n)\right]^{2}\left[G_{\beta_{2}}(n)\right]^{2} H_{3}(n)}\right] \\
& =-2 x^{2}+2 x \text {. }
\end{aligned}
$$

(ii) Using linearity of the operators $S_{n}^{\alpha, \beta}(f(t) ; x)$ and Lemma 2, we have

$$
S_{n}^{\alpha, \beta}(f(t) ; x)=I_{1}^{\alpha, \beta}(n) x^{4}+I_{2}^{\alpha, \beta}(n) x^{3}+I_{3}^{\alpha, \beta}(n) x^{2}+I_{4}^{\alpha, \beta}(n) x+I_{5}^{\alpha, \beta}(n),
$$

where

$$
\begin{aligned}
I_{1}^{\alpha, \beta}(n)= & \frac{n^{8}-6 n^{7}+11 n^{6}-6 n^{5}}{\left[G_{\beta_{1}}(n)\right]^{4} H_{5}(n)}-\frac{4 n^{6}-12 n^{5}-8 n^{4}}{\left[G_{\beta_{1}}(n)\right]^{3} H_{4}(n)}+\frac{6 n^{4}-6 n^{3}}{\left[G_{\beta_{1}}(n)\right]^{2} H_{3}(n)}-\frac{4 n^{2}}{G_{\beta_{1}}(n) H_{2}(n)}+1 \\
I_{2}^{\alpha, \beta}(n)= & \frac{24 n^{7} \alpha_{2}-4 n^{8} \alpha_{2}-44 n^{6} \alpha_{2}+24 n^{5} \alpha_{2}+16 n^{8}-48 n^{7}+32 n^{6}}{\left[G_{\beta_{1}}(n)\right]^{4} G_{\beta_{2}}(n) H_{5}(n)}+\frac{\left.4 n^{7} \alpha_{2}-12 n^{6} \alpha_{2}+8 n^{5} \alpha_{2}\right)}{\left[G_{\beta_{1}}(n)\right]^{4} G_{\beta_{2}}(n) H_{4}(n)} \\
& +\frac{4 n^{4} \alpha_{1}-12 n^{5} \alpha_{1}+8 n^{4} \alpha_{1}}{\left[G_{\beta_{1}}(n)\right]^{4} H_{4}(n)}-\frac{36 n^{6}+72 n^{5} \alpha_{2}-36 n^{7}-72 n^{4} \alpha_{2}}{\left[G_{\beta_{1}}(n)\right]^{3} G_{\beta_{2}}(n) H_{4}(n)}-\frac{12 n^{4} \alpha_{1}-12 n^{3} \alpha_{1}}{\left[G_{\beta_{1}}(n)\right]^{3} H_{3}(n)} \\
& +\frac{24 n^{4}+48 n^{3} \alpha_{2}}{\left[G_{\beta_{1}}(n)\right]^{2} H_{3}(n)}+\frac{12 n^{2} \alpha_{1}}{\left[G_{\beta_{1}}(n)\right]^{2} H_{2}(n)}-\frac{4 n^{2}+8 n \alpha_{2}}{G_{\beta_{1}}(n) G_{\beta_{2}}(n) H_{2}(n)}-\frac{4 \alpha_{1}}{G_{\beta_{1}}(n)} \\
I_{3}^{\alpha, \beta}(n)= & \frac{6 n^{8} \alpha_{2}^{2}-36 n^{7} \alpha_{2}^{2}+66 n^{6} \alpha_{2}^{2}-36 n^{5} \alpha_{2}^{2}-48 n^{8} \alpha_{2}+144 n^{7} \alpha_{2}-96 n^{6} \alpha_{2}+72 n^{8}-72 n^{7}}{\left[G_{\beta_{1}}(n)\right]^{4}\left[G_{\beta_{2}}(n)\right]^{2} H_{5}(n)} \\
& +\frac{-12 n^{7} \alpha_{2}^{2}+36 n^{6} \alpha_{2}^{2}-24 n^{5} \alpha_{2}^{2}+36 n^{7} \alpha_{2}-36 n^{6} \alpha_{2}}{\left[G_{\beta_{1}}(n)\right]^{4}\left[G_{\beta_{2}}(n)\right]^{2} H_{4}(n)}+\frac{6 n^{6} \alpha_{2}^{2}-6 n^{5} \alpha_{2}^{2}}{\left[G_{\beta_{1}}(n)\right]^{4}\left[G_{\beta_{2}}(n)\right]^{2} H_{3}(n)} \\
& +\frac{36 n^{6} \alpha_{1}+72 n^{5} \alpha_{1} \alpha_{2}-36 n^{5} \alpha_{1}-74 n^{4} \alpha_{1} \alpha_{2}}{\left[G_{\beta_{1}}(n)\right]^{4} G_{\beta_{2}}(n) H_{4}(n)}+\frac{6 n^{4} \alpha_{1}^{2}-6 n^{3} \alpha_{1}^{2}}{\left[G_{\beta_{1}}(n)\right]^{4} H_{3}(n)}
\end{aligned}
$$




$$
\begin{aligned}
& +\frac{-36 n^{6} \alpha_{2}-72 n^{6}+216 n^{5} \alpha_{2}-24 n^{5} \alpha_{2}^{2}+264 n^{4} \alpha_{2}^{2}}{\left[G_{\beta_{1}}(n)\right]^{3}\left[G_{\beta_{2}}(n)\right]^{2} H_{4}(n)}-\frac{48 n^{4} \alpha_{1}+96 n^{3} \alpha_{1} \alpha_{2}}{\left[G_{\beta_{1}}(n)\right]^{3} G_{\beta_{2}}(n) H_{3}(n)}-\frac{12 n^{2} \alpha_{1}^{2}}{\left[G_{\beta_{1}}(n)\right]^{3} H_{2}(n)} \\
& +\frac{-12 n^{4} \alpha_{2}-12 n^{4}-12 n^{3} \alpha_{2}^{2}+36 n^{3} \alpha_{2}+36 n^{2} \alpha_{2}^{2}}{\left[G_{\beta_{1}}(n)\right]^{2}\left[G_{\beta_{2}}(n)\right]^{2} H_{3}(n)}+\frac{12 n^{2} \alpha_{1}+24 n \alpha_{1} \alpha_{2}}{\left[G_{\beta_{1}}(n)\right]^{2} G_{\beta_{2}}(n) H_{2}(n)}+\frac{6 \alpha_{1}^{2}}{\left[G_{\beta_{1}}(n)\right]^{2}}, \\
& I_{4}^{\alpha, \beta}(n)=\frac{-4 n^{8} \alpha_{2}^{3}+24 n^{7} \alpha_{2}^{3}-44 n^{6} \alpha_{2}^{3}+24 n^{5} \alpha_{2}^{3}+48 n^{8} \alpha_{2}^{2}-144 n^{7} \alpha_{2}^{2}+96 n^{6}-144 n^{8} \alpha_{2}+144 n^{7} \alpha_{2}+96 n^{4}}{\left[G_{\beta_{1}}(n)\right]^{4}\left[G_{\beta_{2}}(n)\right]^{3} H_{5}(n)} \\
& +\frac{12 n^{7} \alpha_{2}^{3}-36 n^{6} \alpha_{2}^{3}+24 n^{5} \alpha_{2}^{3}-72 n^{7} \alpha_{2}^{2}+72 n^{6} \alpha_{2}^{2}+72 n^{7} \alpha_{2}}{\left[G_{\beta_{1}}(n)\right]^{4}\left[G_{\beta_{2}}(n)\right]^{3} H_{4}(n)}+\frac{-12 n^{6} \alpha_{2}^{3}+12 n^{5} \alpha_{2}^{3}+24 n^{6} \alpha_{2}^{2}}{\left[G_{\beta_{1}}(n)\right]^{4}\left[G_{\beta_{2}}(n)\right]^{3} H_{3}(n)} \\
& +\frac{4 n^{4} \alpha_{2}^{3}}{\left[G_{\beta_{1}}(n)\right]^{4}\left[G_{\beta_{2}}(n)\right]^{3}}+\frac{-36 n^{6} \alpha_{1} \alpha_{2}+72 n^{6} \alpha_{1}+216 n^{5} \alpha_{1} \alpha_{2}-24 n^{5} \alpha_{1} \alpha_{2}^{2}+264 n^{4} \alpha_{1} \alpha_{2}^{2}}{\left[G_{\beta_{1}}(n)\right]^{4}\left[G_{\beta_{2}}(n)\right]^{2} H_{4}(n)} \\
& -\frac{12 n^{6} \alpha_{2}^{2}-48 n^{6} \alpha_{2}+24 n^{6}-96 n^{5} \alpha_{2}^{2}-96 n^{5} \alpha_{2}-96 n^{4} \alpha_{2}^{3}+144 n^{4} \alpha_{2}^{2}+96 n^{3} \alpha_{2}^{3}}{\left[G_{\beta_{1}}(n)\right]^{4}\left[G_{\beta_{2}}(n)\right]^{3} H_{4}(n)} \\
& -\frac{-24 n^{4} \alpha_{1}^{2} \alpha_{2}+24 n^{4} \alpha_{1}-24 n^{3} \alpha_{1} \alpha_{2}^{2}-72 n^{3} \alpha_{1} \alpha_{2}+72 n^{2} \alpha_{1} \alpha_{2}^{2}}{\left[G_{\beta_{1}}(n)\right]^{4}\left[G_{\beta_{2}}(n)\right]^{2} H_{3}(n)}+\frac{24 n^{4} \alpha_{1}^{2}+48 n^{3} \alpha_{1}^{2} \alpha_{2}}{\left[G_{\beta_{1}}(n)\right]^{4} G_{\beta_{2}}(n) H_{3}(n)} \\
& +\frac{3 n^{2} \alpha_{1}^{2}+6 n \alpha_{1}^{2} \alpha_{2}}{\left[G_{\beta_{1}}(n)\right]^{3} G_{\beta_{2}}(n) H_{2}(n)}+\frac{4 n^{2} \alpha_{1}^{3}}{\left[G_{\beta_{1}}(n)\right]^{4} H_{2}(n)}+\frac{\alpha_{1}^{3}}{\left[G_{\beta_{1}}(n)\right]^{3}}, \\
& I_{5}^{\alpha, \beta}(n)=\frac{n^{8} \alpha_{2}^{4}-6 n^{7} \alpha_{2}^{4}+11 n^{6} \alpha_{2}^{4}-6 n^{5} \alpha_{2}^{4}-16 n^{8} \alpha_{2}^{3}+48 n^{7} \alpha_{2}^{3}-32 n^{6} \alpha_{2}^{3}+72 n^{8} \alpha_{2}^{2}-72 n^{7} \alpha_{2}^{2}+24 n^{6}-96 n^{8} \alpha_{2}}{\left[G_{\beta_{1}}(n)\right]^{4}\left[G_{\beta_{2}}(n)\right]^{4} H_{5}(n)} \\
& +\frac{-4 n^{7} \alpha_{2}^{4}+12 n^{6} \alpha_{2}^{4}-8 n^{5} \alpha_{2}^{4}+36 n^{7} \alpha_{2}^{3}-36 n^{6} \alpha_{2}^{3}-72 n^{7} \alpha_{2}^{2}+24 n^{7} \alpha_{2}}{\left[G_{\beta_{1}}(n)\right]^{4}\left[G_{\beta_{2}}(n)\right]^{4} H_{4}(n)}+\frac{6 n^{2} \alpha_{2}^{4}-6 n^{5} \alpha_{2}^{4}-24 n^{2} \alpha_{2}^{3}+12 n^{6} \alpha_{2}^{2}}{\left[G_{\beta_{1}}(n)\right]^{4}\left[G_{\beta_{2}}(n)\right]^{4} H_{3}(n)} \\
& +\frac{12 n^{6} \alpha_{1} \alpha_{2}^{2}-48 n^{6} \alpha_{1} \alpha_{2}+24 n^{6} \alpha_{1}-96 n^{5} \alpha_{1} \alpha_{2}^{2}-96 n^{5} \alpha_{1} \alpha_{2}-96 n^{4} \alpha_{1} \alpha_{2}^{3}+144 n^{4} \alpha_{1} \alpha_{2}^{2}+96 n^{3} \alpha_{1} \alpha_{2}^{3}}{\left[G_{\beta_{1}}(n)\right]^{4}\left[G_{\beta_{2}}(n)\right]^{3} H_{4}(n)} \\
& +\frac{\alpha_{1}^{4}}{\left[G_{\beta_{1}}(n)\right]^{4}}+\frac{-12 n^{4} \alpha_{1}^{2} \alpha_{2}+12 \alpha_{1}^{2} n^{4}-12 n^{3} \alpha_{1}^{2} \alpha_{2}^{2}+36 n^{2} \alpha_{1}^{2} \alpha_{2}+36 n^{2} \alpha_{1}^{2} \alpha_{2}^{2}}{\left[G_{\beta_{1}}(n)\right]^{2}\left[G_{\beta_{2}}(n)\right]^{2} H_{3}(n)}+\frac{4 n^{2} \alpha_{1}^{3}+8 n \alpha_{1}^{3} \alpha_{2}}{\left[G_{\beta_{1}}(n)\right]^{4} G_{\beta_{2}}(n) H_{2}(n)} \\
& +\frac{\left(4 n^{4} \alpha_{2}^{3}-3 n^{4} \alpha_{2}^{4}\right)}{\left[G_{\beta_{1}}(n)\right]^{4}\left[G_{\beta_{2}}(n)\right]^{4}}
\end{aligned}
$$

It is obvious that

$$
\lim _{n \rightarrow \infty} n^{2}\left\{I_{4}^{\alpha, \beta}(n)+I_{5}^{\alpha, \beta}(n)\right\}=0 .
$$

Therefore, we have

$$
\lim _{n \rightarrow \infty} n^{2}\left\{I_{1}^{\alpha, \beta}(n)+I_{2}^{\alpha, \beta}(n)+I_{3}^{\alpha, \beta}(n)\right\}=28 x^{4}-24 x^{3}+\left(12 \alpha_{2}+12\right) x^{2} .
$$

Adding the limits (7) and (8), we have the limits (6).

In [6], Gadzhiev proved the weighted Korovkin-type theorem. We give the Gadzhiev's results in weighted spaces. Let $\mu(x)=1+x^{2} \cdot B_{\mu}[0, \infty)$ denotes the set of all functions from $[0, \infty)$ to $\mathbb{R}$, satisfying the growth condition $|f(x)| \leq N_{f} \mu(x)$. In this inequality, $N_{f}$ is a constant depending only on $f . B_{\mu}[0, \infty)$ is a normed 
space with a norm $\|f\|_{\mu}=\sup \left\{\frac{|f(x)|}{\mu(x)} ; x \in \mathbb{R}\right\} . C_{\mu}^{*}[0, \infty)$ denotes the subspace of continuos functions in $B_{\mu}[0, \infty)$ for which $\lim _{|x| \rightarrow \infty} \frac{|f(x)|}{\mu(x)}$ exists finitely.

And now we give a Voronovskaja-type theorem for $S_{n}^{\alpha, \beta}(f(t), x)$ operators.

Theorem 1. For any $f \in C_{\mu}^{*}[0, \infty)$ such that $f^{\prime}, f^{\prime \prime} \in C_{\mu}^{*}[0, \infty)$. We get the following limit $\lim _{n \rightarrow \infty} n\left(S_{n}^{\alpha, \beta}(f(t) ; x)-f(x)\right)=\left(-x^{2}+x\right) f^{\prime \prime}(x)+\left(\left(-2-\beta_{1}\right) x+1+\alpha_{2}\right) f^{\prime}(x)$.

Proof. Using Taylor's expansion of $f$, we have

$f(t)=f(x)+f^{\prime}(x)(t-x)+\frac{1}{2} f^{\prime \prime}(x)(t-x)^{2}+\rho(t, x)(t-x)^{2}$,

where $\rho(t, x) \rightarrow 0$ as $t \rightarrow x$. From linearity of the operators $S_{n}^{\alpha, \beta}(f(t), x)$, we get

$S_{n}^{\alpha, \beta}(f(t) ; x)=f(x)+f^{\prime}(x) S_{n}^{\alpha, \beta}(t-x)+\frac{1}{2} f^{\prime \prime}(x) S_{n}^{\alpha, \beta}\left((t-x)^{2} ; x\right)$

$$
+S_{n}^{\alpha, \beta}\left(\rho(t, x)(t-x)^{2}\right) .
$$

Thanks to Lemma 2, we obtain the following operators by making the necessary process,

$$
\begin{aligned}
S_{n}^{\alpha, \beta}(f(t) ; x)= & f(x)+f^{\prime}(x)\left\{\frac{-n\left(2+\beta_{1}\right)-2 \beta_{2}}{G_{\beta_{1}}(n) H_{4}(n)} x+\frac{n^{2}+2 n \alpha_{2}}{G_{\beta_{1}}(n) G_{\beta_{2}}(n) H_{2}(n)}+\frac{\alpha_{2}}{G_{\beta_{1}}(n)}\right\} \\
& +\frac{1}{2} f^{\prime \prime}(x)\left[\left\{\frac{-2 n^{3}+n^{2}\left(6+\beta_{1}{ }^{2}+4 \beta_{1}\right)+n\left(5 \beta_{1}{ }^{2}+12 \beta_{1}\right)+6 \beta_{1}{ }^{2}}{\left[G_{\beta_{1}}(n)\right]^{2} H_{3}(n)}\right\} x^{2}\right. \\
& +\left\{\frac{2 n^{4}+n^{3}\left(4 \alpha_{2}+4 \alpha_{1}-2 \alpha_{1} \beta_{1}-2 \beta_{1}-6\right)}{\left[G_{\beta_{1}}(n)\right]^{2}\left[G_{\beta_{2}}(n)\right]^{2} H_{3}(n)}\right. \\
& -\frac{n^{2}\left(12 \alpha_{1}+12 \alpha_{2}+10 \alpha_{1} \beta_{1}+4 \alpha_{1} \beta_{2}+4 \alpha_{2} \beta_{1}+2 \alpha_{1} \beta_{1} \beta_{2}+6 \beta_{1}\right)}{\left[G_{\beta_{1}}(n)\right]^{2}\left[G_{\beta_{2}}(n)\right]^{2} H_{3}(n)} \\
& \left.+\frac{12 \alpha_{1} \beta_{1}+12 \alpha_{1} \beta_{2}+12 \alpha_{2} \beta_{1}+10 \alpha_{1} \beta_{1} \beta_{2}-12 \alpha_{1} \beta_{1} \beta_{2}}{\left[G_{\beta_{1}}(n)\right]^{2}\left[G_{\beta_{2}}(n)\right]^{2} H_{3}(n)}\right\} x \\
& +\frac{n^{4}\left(4 \alpha_{2}^{2}-2 \alpha_{2}+\alpha_{1}^{2}+2 \alpha_{1}+2\right)+n^{3}\left(10 \alpha_{2}^{2}+6 \alpha_{2}+5 \alpha_{1}^{2}+6 \alpha_{1}+5 \alpha_{1}^{2} \beta_{2}+2 \alpha_{1} \alpha_{2}+2 \alpha_{1} \beta_{2}\right)}{\left[G_{\beta_{1}}(n)\right]^{2}\left[G_{\beta_{2}}(n)\right]^{2} H_{3}(n)} \\
& +\frac{2\left(6 \alpha_{2}^{2}+6 \alpha_{1}^{2}+10 \alpha_{1}^{2} \beta_{2}+\alpha_{1}^{2} \beta_{2}{ }^{2}+12 \alpha_{1} \alpha_{2}+6 \alpha_{1} \beta_{2}+4 \alpha_{1} \alpha_{2} \beta_{2}\right)}{\left[G_{\beta_{1}}(n)\right]^{2}\left[G_{\beta_{2}}(n)\right]^{2} H_{3}(n)} \\
& \left.+\frac{n\left(5 \alpha_{1}^{2} \beta_{2}{ }^{2}+12 \alpha_{1}^{2} \beta_{2}+12 \alpha_{1} \alpha_{2} \beta_{2}\right)+6 \alpha_{1}^{2} \beta_{2}{ }^{2} n}{\left[G_{\beta_{1}}(n)\right]^{2}\left[G_{\beta_{2}}(n)\right]^{2} H_{3}(n)}\right]+S_{n}^{\alpha, \beta}\left(\rho(t, x)(t-x)^{2} ; x\right) .
\end{aligned}
$$

Applying Cauchy-Schwarz inequality to the last term of (9) we obtain

$$
n S_{n}^{\alpha, \beta}\left(\rho(t, x)(t-x)^{2} ; x\right) \leq S_{n}^{\alpha, \beta}\left(\rho(t, x)^{2} ; x\right)^{\frac{1}{2}}\left(n^{2} S_{n}^{\alpha, \beta}\left((t-x)^{4} ; x\right)\right)^{\frac{1}{2}}
$$

and 


$$
\lim _{n \rightarrow \infty} n S_{n}^{\alpha, \beta}\left(\rho(t, x)(t-x)^{2} ; x\right)
$$

$$
\leq\left(\lim _{n \rightarrow \infty} S_{n}^{\alpha, \beta}\left(\rho(t, x)^{2} ; x\right)\right)^{\frac{1}{2}}\left(\lim _{n \rightarrow \infty} n^{2} S_{n}^{\alpha, \beta}\left((t-x)^{4} ; x\right)\right)^{\frac{1}{2}} .
$$

Inasmuch as $\lim _{n \rightarrow \infty} S_{n}^{\alpha, \beta}\left(\rho(t, x)^{2} ; x\right)=0$ and from (ii) of Lemma 3, $\lim _{n \rightarrow \infty} n^{2} S_{n}^{\alpha, \beta}\left((t-x)^{4} ; x\right)$ is finite.

Therefore, we get

$$
\begin{aligned}
& \lim _{n \rightarrow \infty} n S_{n}^{\alpha, \beta}\left(\rho(t, x)(t-x)^{2} ; x\right)=0, \\
& \lim _{n \rightarrow \infty} n\left(S_{n}^{\alpha, \beta}(f(t) ; x)-f(x)\right)=\left(\left(-2-\beta_{1}\right) x+1+\alpha_{2}\right) f^{\prime}(x)+\left(-x^{2}+x\right) f^{\prime \prime}(x) .
\end{aligned}
$$

Hence, the theorem is proved.

\section{ACKNOWLEDGEMENTS}

The authors would like to thank the referees for the careful reading of this paper and for their valuable suggestions to improve the paper.

\section{CONFLICTS OF INTEREST}

No conflict of interest was declared by the authors.

\section{REFERENCES}

[1] Bernstein, S. N., "Demonstration du theoreme de Weierstrass fondee sur le calcul de probabilities", Commun. Soc. Math. Kharkow, 13(2): 1-2, (1912).

[2] Durrmeyer, J. L., "Une Formule D’inversion de la transformée de Laplace: Aplications a la théorie des moments, Thése de 3e cycle", Faculté des Sciences de I'Université de Paris, (1967).

[3] Gadhziev, A. D. and Chorbanalizadeh, A. M., "Approximation properties of a new type BernsteinStancu polynomials of one and two variables”, Appl. Math. Comput., 216: 890-901, (2010).

[4] Stancu, D. D., "Approximation of functions by a new class of linear polynomial operators", Rev. Roum. Math. Pures Appl., 13: 1173-1194, (1968).

[5] Dong, L. X. and Yu, D. S., "Pointwise Approximation by a Durrmeyer Variant of Bernstein-Stancu Operators", J. Inequal Appl., 2017, 28, (2017).

[6] Gadhziev, A. D., "Theorems of the type of P.P. Korovkin type theorems", Math. Zametki, 20(5): 781786, (1976). (English Translation, Math. Notes, 20(5/6): 996-998, (1976).

[7] Gupta, V. and Duman, O., "Bernstein-Durrmeyer type operators preserving linear function", Matematicki Vesnik, 62(4): 259-264, (2010).

[8] Tasdelen, F., Bascanbaz-Tunca, G. and Erencin, A., "On a new type Bernstein-Stancu operators", Fasc. Math., 48: 119-128, (2012). 
[9] Acar, T., Aral, A. and Gupta, V., "On approximation properties of a new type Bernstein-Durrmeyer operators”, Math. Slovaca., 65: 1107-1122, (2015).

[10] Icoz, G., "A Kantorovich variant of a new type Bernstein Stancu polynomials”, Appl. Math. Comput., 218: 8552-8560, (2012).

[11] Wang, M. L., Yu, D. S. and Zhou, P., "On the approximation by operators of Bernstein-Stancu types", Appl. Math. Comput., 246: 79-87, (2014). 